Research Article

\title{
Nonlinear Stochastic Optimal Control Using Piezoelectric Stack Inertial Actuator
}

\author{
Q. F. Lü, X. F. Wang, K. Lu, and R. H. Huan \\ Department of Mechanics, State Key Laboratory of Fluid Power and Mechatronic Systems, \\ Key Laboratory of Soft Machines and Smart Devices of Zhejiang Province, Zhejiang University, Hangzhou 310027, China
}

Correspondence should be addressed to R. H. Huan; rhhuan@zju.edu.cn

Received 7 December 2019; Revised 17 March 2020; Accepted 12 May 2020; Published 18 August 2020

Academic Editor: Evgeny Petrov

Copyright $(92020$ Q. F. Lü et al. This is an open access article distributed under the Creative Commons Attribution License, which permits unrestricted use, distribution, and reproduction in any medium, provided the original work is properly cited.

An optimal control strategy for the random vibration reduction of nonlinear structures using piezoelectric stack inertial actuator is proposed. First, the dynamic model of the nonlinear structure considering the dynamics of a piezoelectric stack inertial actuator is established, and the motion equation of the coupled system is described by a quasi-non-integrableHamiltonian system. Then, using the stochastic averaging method, this quasi-non-integrable-Hamiltonian system is reduced to a one-dimensional averaged system for total energy. The optimal control law is determined by establishing and solving the dynamic programming equation. The proposed control law is analytical and can be fully executed by a piezoelectric stack inertial actuator. The responses of optimally controlled and uncontrolled systems are obtained by solving the Fokker-Planck-Kolmogorov (FPK) equation to evaluate the control effectiveness of the proposed strategy. Numerical results show that our proposed control strategy is effective for random vibration reduction of the nonlinear structures using piezoelectric stack inertial actuator, and the theoretical method is verified by comparing with the simulation results.

\section{Introduction}

Piezoelectric stack actuators have been widely used in vibration control of mechanical structures due to their fast response and high precision, such as aerospace, precision machining, biomedical engineering, and semiconductor manufacturing [1-4]. Generally, there are two basic approaches when a piezoelectric stack actuator is used as an actuator. One is the direct actuator, where one side of the piezoelectric stack is fixed and the other is bonded to the structure. Although this kind of actuator has large output force and an easily determined control law, it could bring new excitation sources to the structure. The other way is to use as an inertial actuator, where one side is combined with an inertial mass and the other side is bonded to a structure. The inertial mass can effectively isolate unnecessary interference and also can protect the pressure sensor from being damaged by excessive force [5]. This way is commonly used and has been applied by many scholars in some different areas. For example, Choi et al. [6] applied a piezoelectric stack inertial actuator to the vibration control of simply supported beam at both ends and achieved good control effectiveness. Li et al. [7] applied a piezoelectric stack actuator to an active shaft transverse vibration control system with large reduction of housing vibrations. Zhao et al. [8] used a piezoelectric rotary inertia actuator to control the vibration of the rotating structure, which effectively reduced the noise propagation of the structure. Shao et al. [9] proposed a new type of inertial piezoelectric actuator which has a miniaturization structure and dynamic performance of high precision and high load capacity. Wang et al. [10] obtained an actuator with stable linear motion performance using integrated piezoelectric vibrator and MRF control structure. The traditional piezoelectric inertia actuator can only bear the force in the axial direction. For this reason, Konstanzer et al. [11] studied and designed an actuator that can bear the bending stress, which greatly improved the control effect with the condition of large force and low 
frequency vibration. Introducing the modal $\mathrm{H}_{2}$ norm and change rate of natural frequencies, Lu et al. [12] proposed an optimal placement criterion for piezoelectric actuators. With this criterion, the piezoelectric smart SFM system has a better single modal controllability and observability and has a good result on the vibration suppression.

Through the survey of these literatures, it can be found that most of the studies on vibration control using piezoelectric stack inertial actuator mentioned above are limited to the study of the dynamic characteristics of the actuator itself or the vibration control of linear structure under the action of deterministic load. Far less is known about the control of random vibration, especially nonlinear random vibration. Thus, the development of a control strategy for a nonlinear stochastic system using a piezoelectric stack inertial actuator is much deserving, and that is the motivation of the present paper.

In the present paper, an optimal control problem for a strong nonlinear and stochastically excited structure with a piezoelectric stack inertial actuator is investigated. Our original contributions are highlighted as follows: the dynamic model of the nonlinear structure considering random excitation and the dynamics of a piezoelectric stack inertial actuator is established; the control problem is firstly investigated in the Hamiltonian frame, which makes the stochastic averaging method for the quasi-Hamiltonian system available for dimension reduction; the proposed optimal control law, which can be fully executed by a piezoelectric stack inertial actuator, is robust and effective in reduction of random vibration.

\section{Mathematical Modeling of the Coupled System}

Figure 1 presents schematic configuration of the piezoelectric stack inertial actuator consisting of an inertial mass and a piezoelectric stack. The electromechanical behavior of the piezoelectric actuator can be expressed as follows [13]:

$$
\begin{aligned}
& S_{3}=s_{33}^{E} T_{3}+d_{33} E_{3}, \\
& D_{3}=d_{33} T_{3}+\varepsilon_{33}^{T} E_{3},
\end{aligned}
$$

where $S$ is the strain, $D$ is the electrical displacement, $T$ is the stress, $E$ is the electric field, $d$ is the piezoelectric material constant, $\varepsilon^{E}$ is the elastic compliance, and $\varepsilon^{T}$ is the permittivity at a constant stress. Thus, the dynamic behavior of $n$ piezoceramic layers can be derived as follows:

$$
\begin{aligned}
F_{p}(t) & =A T_{3}=\frac{A}{s_{33}} S_{33}-\frac{A d_{33}}{s_{33}} E_{3} \\
& =\frac{A}{s_{33}} \frac{\delta(t)}{l}-\frac{n A d_{33}}{s_{33}} \frac{V_{p}(t)}{l} \\
& =K_{p} \delta(t)-\alpha_{p} V_{p}(t) \\
& =K_{p} \delta(t)-F_{a}(t),
\end{aligned}
$$

where $F_{p}(t)$ is the load of the piezoelectric stack inertial actuator, $A$ is the cross-sectional area of the piezoelectric stack, $l$ and $\delta(t)$ are the length and deformation of the piezoelectric stack, $F_{a}(t)$ is the force generated by voltage $V_{p}(t), K_{p}$ is the stiffness of the spring, and $\alpha_{p}$ is the proportional constant. Considering the damping in piezoelectric stack, the motion equation of the mechanical model in Figure 1(b) can be established:

$$
m \ddot{x}+c_{p} \dot{x}+k_{p} x+\alpha_{p} V_{p}(t)=0 .
$$

Here, we use this inertial actuator for vibration control of a nonlinear structure. The coupled system is shown in Figure 2. In this figure, $k_{p}$ and $c_{p}$ denote, respectively, the stiffness and damping of the piezoelectric stack actuator; $m$ and $M$ are the mass of the inertial actuator and the mass of vibration structure, respectively; $x$ is the displacement of the vibration structure; $y$ is the displacement of inertial mass; and $F$ is the active control force exerted by voltage. $Z(t)$ is random disturbance of the base. Then, the motion equation of the coupled system can be established:

$$
\begin{aligned}
& M \ddot{x}+c[\dot{x}-\dot{Z}(t)]+k[x-Z(t)]+k_{3}[x-Z(t)]^{3}-k_{p}(y-x)-c_{p}(\dot{y}-\dot{x})=-F, \\
& m \ddot{y}+c_{p}[\dot{y}-\dot{x}]+k_{p}[y-x]=F .
\end{aligned}
$$

System (4) is a two-degree-of-freedom, strong nonlinear, stochastic excited, and controlled system.

\section{Partial Averaging of the Controlled System}

Introduce the transformation

$$
\begin{aligned}
& \bar{x}=x-Z(t), \\
& \bar{y}=y-x .
\end{aligned}
$$

Substituting (5) to (4) yields 


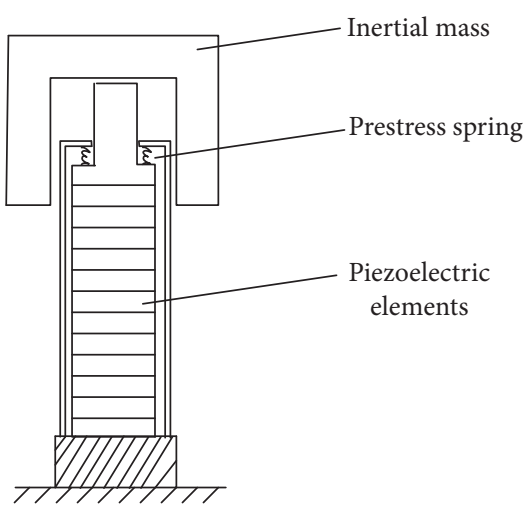

(a)

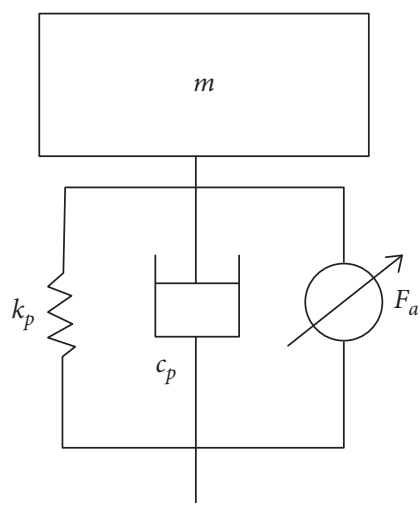

(b)

FIgURE 1: Configuration and model of piezoelectric stack inertial actuator. (a) Schematic configuration. (b) Mechanical model.

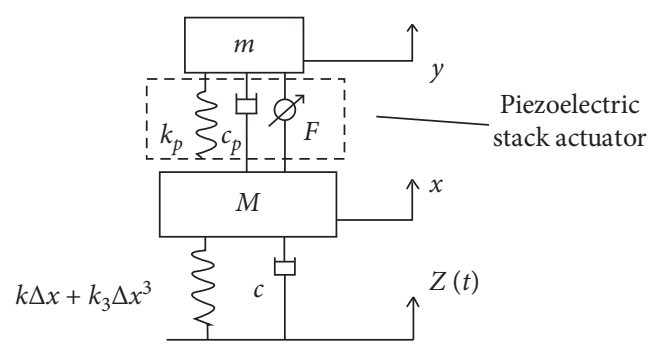

FIGURE 2: Mechanical model of the coupled system.

$$
\begin{aligned}
& M \ddot{\bar{x}}+c \dot{\bar{x}}-c_{p} \dot{\bar{y}}+k \bar{x}+k_{3} \bar{x}^{3}-k_{p} \bar{y}=-F_{u}-M \ddot{Z}(t), \\
& m \ddot{\bar{y}}+c_{p}\left(1+\frac{m}{M}\right) \dot{\bar{y}}-\frac{m}{M} c \dot{\bar{x}}-\frac{m}{M}\left(k \bar{x}+k_{3} \bar{x}^{3}\right)+k_{p}\left(1+\frac{m}{M}\right) \bar{y}=\left(1+\frac{m}{M}\right) F_{u} .
\end{aligned}
$$

Introduce the generalized displacement

$$
\begin{aligned}
& q_{1}=\bar{x}, \\
& p_{1}=M \dot{\bar{x}}, \\
& q_{2}=\bar{x}+\bar{y}, \\
& p_{2}=m(\dot{\bar{x}}
\end{aligned}
$$

and system (4) can be rewritten as

$$
\begin{aligned}
& \dot{q}_{1}=\frac{\partial H}{\partial p_{1}}, \\
& \dot{p}_{1}=-\frac{\partial H}{\partial q_{1}}-\left(c+c_{p}\right) \frac{\partial H}{\partial p_{1}}+c_{p} \frac{\partial H}{\partial p_{2}}-F-M \ddot{Z}(t), \\
& \dot{q}_{2}=\frac{\partial H}{\partial p_{2}}, \\
& \dot{p}_{2}=-\frac{\partial H}{\partial q_{2}}-c_{p}\left(\frac{\partial H}{\partial p_{2}}-\frac{\partial H}{\partial p_{1}}\right)+-m \ddot{Z}(t),
\end{aligned}
$$

where $\ddot{Z}(t)$ is acceleration of the base, which is assumed to be a zero-mean Gaussian white noise with correlation function $E\left[\omega_{k}(t) \times \omega_{l}(t+\tau)\right]=2 D_{k l} \delta(\tau)$. System (8) is called a quasi-Hamiltonian system. The Hamiltonian function associated with equation (8) is

$$
H=\frac{1}{2} \frac{p_{1}^{2}}{M}+\frac{1}{2} \frac{p_{2}^{2}}{m}+\frac{1}{2} k q_{1}^{2}+\frac{1}{4} k_{3} q_{1}^{4}+\frac{1}{2} k_{p}\left(q_{2}-q_{1}\right)^{2} .
$$

Obviously, $H$ is the only first integral, which indicates that system (5) is a quasi-non-integrable-Hamiltonian system [14]. In most engineering applications, the Hamiltonian function $H$ denotes the total vibration energy of the structures.

Theoretically, by adding Wong-Zakai terms, system (8) can be converted into the following Itô form:

$$
\begin{aligned}
\mathrm{d} Q_{1} & =\frac{\partial H}{\partial P_{1}} \mathrm{~d} t, \\
\mathrm{~d} P_{1} & =\left[-\frac{\partial H}{\partial Q_{1}}-m_{11} \frac{\partial H}{\partial P_{1}}-m_{12} \frac{\partial H}{\partial P_{2}}-u\right] \mathrm{d} t+\sigma_{1} \mathrm{~d} B(t), \\
\mathrm{d} Q_{2} & =\frac{\partial H}{\partial P_{2}} \mathrm{~d} t, \\
\mathrm{~d} P_{2} & =\left[-\frac{\partial H}{\partial Q_{2}}-m_{21} \frac{\partial H}{\partial P_{1}}-m_{22} \frac{\partial H}{\partial P_{2}}+u\right] \mathrm{d} t+\sigma_{2} \mathrm{~d} B(t),
\end{aligned}
$$


where $\sigma_{1}^{2}=2 D M^{2}, \sigma_{2}^{2}=2 D m^{2}, m_{11}=c+c_{p}, m_{12}=-c_{p}$, $m_{21}=-c_{p}, m_{22}=c_{p}, u_{1}=-F, u_{2}=F$, and $B(t)$ is the standard Wiener process. Then, the stochastic averaging method for quasi-non-integrable-Hamiltonian system is applied to system (10), and the averaged Itô equation for $H$ can be obtained as follows:

$$
\mathrm{d} H=\left[\bar{m}(H)+\left\langle u\left(-\frac{\partial H}{\partial P_{1}}+\frac{\partial H}{\partial P_{2}}\right)\right\rangle\right] \mathrm{d} t+\bar{\sigma}(H) \mathrm{d} B(t),
$$

where $\bar{m}(H)$ and $\bar{\sigma}(H)$ are drift and diffusion coefficients, and

$$
\begin{aligned}
\bar{m}(H) & =\frac{1}{T(H)} \int_{\Omega}\left[\left(\frac{\left(-\sum_{i, j=1}^{2} m_{i j}\left(\partial H / \partial p_{i}\right)\left(\partial H / \partial p_{j}\right)+\sum_{i, j=1}^{2}(1 / 2) \sigma_{i} \sigma_{j}\left(\partial^{2} H / \partial p_{i} \partial p_{j}\right)\right)}{\left(\partial H / \partial p_{1}\right)}\right)\right] \mathrm{d} q_{1} \mathrm{~d} q_{2} \mathrm{~d} p_{2} \\
& =\frac{1}{T(H)} \int_{\Omega}\left[\left(\frac{-m_{11}\left(\partial H / \partial p_{1}\right)\left(\partial H / \partial p_{1}\right)-2 m_{12}\left(\partial H / \partial p_{1}\right)\left(\partial H / \partial p_{2}\right)-m_{22}\left(\partial H / \partial p_{2}\right)\left(\partial H / \partial p_{2}\right)+(1 / 2) \sigma_{1}^{2}\left(\partial^{2} H / \partial^{2} p_{1}\right)+\sigma_{1} \sigma_{2}\left(\partial^{2} H / \partial p_{1} \partial p_{2}\right)+(1 / 2) \sigma_{2}^{2}\left(\partial^{2} H / \partial^{2} p_{2}\right)}{\left(\partial H / \partial p_{1}\right)}\right)\right] \mathrm{d} q_{1} \mathrm{~d} q_{2} \mathrm{~d} p_{2}, \\
\bar{\sigma}^{2}(H) & =\frac{1}{T(H)} \int_{\Omega}\left[\left(\frac{\sum_{i, j=1}^{2} \sigma_{i} \sigma_{j}\left(\partial H / \partial p_{i}\right)\left(\partial H / \partial p_{j}\right)}{\left(\partial H / \partial p_{1}\right)}\right)\right] \mathrm{d} q_{1} \mathrm{~d} q_{2} \mathrm{~d} p_{2} \\
& =\frac{1}{T(H)} \int_{\Omega}\left[\left(\frac{\sigma_{1} \sigma_{1}\left(\partial H / \partial p_{1}\right)\left(\partial H / \partial p_{1}\right)+2 \sigma_{1} \sigma_{2}\left(\partial H / \partial p_{1}\right)\left(\partial H / \partial p_{2}\right)+\sigma_{2} \sigma_{2}\left(\partial H / \partial p_{2}\right)\left(\partial H / \partial p_{2}\right)}{\left(\partial H / \partial p_{1}\right)}\right)\right] \mathrm{d} q_{1} \mathrm{~d} q_{2} \mathrm{~d} p_{2} \\
\langle\cdot\rangle & =\frac{1}{T(H)} \int_{\Omega}\left(\frac{\cdot}{\left(\partial H / \partial p_{1}\right)}\right) \mathrm{d} q_{1} \mathrm{~d} q_{2} \mathrm{~d} p_{2} \\
T(H) & =\int_{\Omega}\left(\frac{1}{\left(\partial H / \partial p_{1}\right)}\right) \mathrm{d} q_{1} \mathrm{~d} q_{2} \mathrm{~d} p_{2} \\
\Omega & =\left\{\left(q_{1}, q_{2}, p_{2}\right) \mid H\left(q_{1}, q_{2}, 0, p_{2}\right) \leq H\right\} .
\end{aligned}
$$

To complete the integration above, make the following polar coordinate transformation:

$$
\begin{aligned}
& q_{1}=\left(\frac{R}{\omega_{1}}\right) \cos \theta, \\
& q_{2}=\left(\frac{R}{\omega_{2}}\right) \sin \theta,
\end{aligned}
$$

and the coefficients $\bar{m}(H), \bar{\sigma}^{2}(H)$, and $T(H)$ can be rewritten as

$$
\begin{aligned}
\bar{m}(H)= & \frac{2 \pi}{T(H) \omega_{1} \omega_{2}} \int_{0}^{2 \pi}\left[\frac{1}{2}\left(\lambda_{1} H+\lambda_{2} H+\lambda_{3}\right) R^{2}\right. \\
& \left.-\left(\lambda_{1}+\lambda_{2}\right) A(H, \theta)\right] \mathrm{d} \theta, \\
\bar{\sigma}^{2}(H)= & \frac{2 \pi}{T(H) \omega_{1} \omega_{2}} \int_{0}^{2 \pi}\left[\frac{1}{2}\left(\lambda_{4} H+\lambda_{5} H\right) R^{2}\right. \\
& \left.-\left(\lambda_{4}+\lambda_{5}\right) A(H, \theta)\right] \mathrm{d} \theta, \\
T(H)= & \frac{2 \pi \sqrt{M m}}{\omega_{1} \omega_{2}} \int_{0}^{\pi} R^{2} \mathrm{~d} \theta,
\end{aligned}
$$

where $\quad \lambda_{1}=-m_{11} \sqrt{m / M}, \quad \lambda_{2}=-m_{22} \sqrt{M / m}, \quad \lambda_{3}=(1 / 2)$ $\left(\sigma_{1}^{2} \sqrt{m / M}+\sigma_{2}^{2} \sqrt{M / m}\right), \quad \lambda_{4}=\sigma_{1}^{2} \sqrt{m / M}, \quad \lambda_{5}=\sigma_{2}^{2} \sqrt{M / m}$, and

$$
\begin{aligned}
A(H, \theta)= & \frac{1}{8} k \frac{\cos ^{2} \theta}{\omega_{1}^{2}} R^{4}+\frac{1}{24} k_{3} \frac{\cos ^{4} \theta}{\omega_{1}^{4}} R^{6} \\
& +\frac{1}{8} k_{p}\left(\frac{\cos \theta}{\omega_{1}}-\frac{\sin \theta}{\omega_{2}}\right)^{2} R^{4} .
\end{aligned}
$$

$R^{2}$ in equations (14) and (15) is the solution of following equation:

$$
H-\frac{1}{2} k \frac{R^{2}}{\omega_{1}^{2}} \cos ^{2} \theta-\frac{1}{4} k_{3} \frac{R^{4}}{\omega_{1}^{4}} \cos ^{4} \theta-\frac{1}{2} k_{p} R^{2}\left(\frac{\sin \theta}{\omega_{2}}-\frac{\cos \theta}{\omega_{1}}\right)^{2}=0 .
$$

\section{Optimal Control Law}

Usually, the following performance of index is used for vibration control problem:

$$
J(\mathbf{u})=E\left[\int_{t_{o}}^{t_{f}}(\mathbf{X}, \mathbf{u}, t) \mathrm{d} t+g\left(\mathbf{X}\left(t_{f}\right)\right)\right] .
$$

Consistent with the averaged equation (11), the averaged form for the proposed performance is obtained as [15]

$$
J(u)=\lim _{t_{f} \longrightarrow \infty} \frac{1}{t_{f}} \int_{0}^{t_{f}} f(H(s),\langle u(s)\rangle) \mathrm{d} s+g\left(H\left(t_{f}\right)\right) .
$$




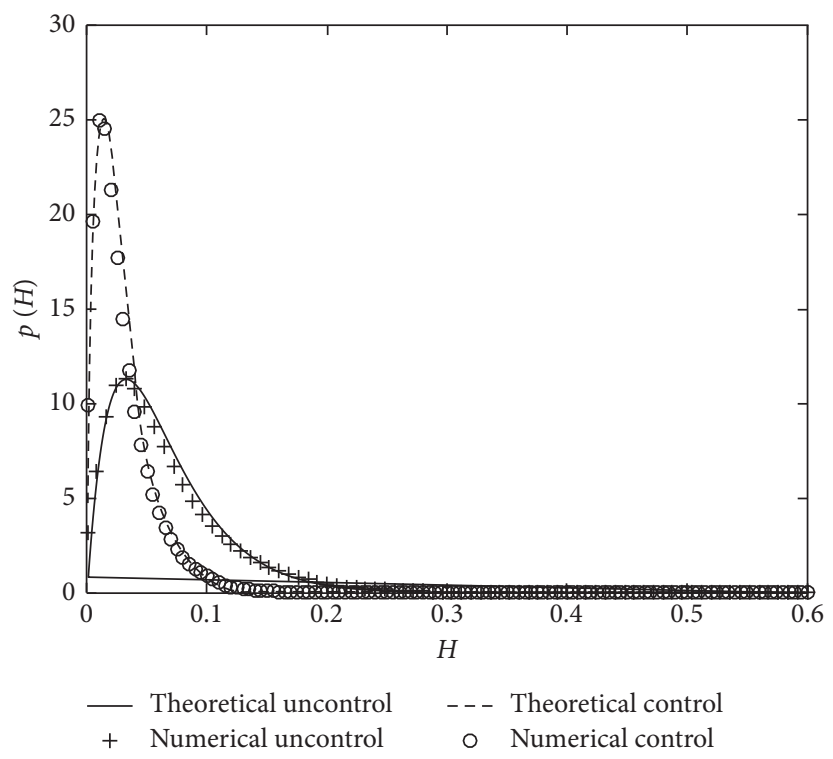

FIGURE 3: Stationary probability density $p(H)$ of controlled and uncontrolled system (10).

Equations (11) and (18) constitute the mathematical formulation of the optimal control problem of the partially averaged quasi-non-integrable-Hamiltonian system with piezoelectric stack inertial actuator.
According to the dynamic programming principle, the dynamic programming equation is established as

$$
\frac{\partial V}{\partial t}=-\inf _{u}\left\{\frac{1}{2} \bar{\sigma}^{2}(H) \frac{\mathrm{d}^{2} H}{\mathrm{~d} H^{2}}+\left[\bar{m}(H)+\left\langle u\left(-\frac{\partial H}{\partial P_{1}}+\frac{\partial H}{\partial P_{2}}\right)\right\rangle\right] \frac{\mathrm{d} V}{\mathrm{~d} H}+f(H,\langle u\rangle)\right\},
$$

where $V(H, t)=g\left(H\left(t_{f}\right)\right)$ is the terminal condition. The necessary condition for minimizing the right-hand side of equation (19) is

$$
\frac{\partial}{\partial u}\left[\left\langle u\left(-\frac{\partial H}{\partial P_{1}}+\frac{\partial H}{\partial P_{2}}\right)\right\rangle \frac{\mathrm{d} V}{\mathrm{~d} H}+f(H,\langle u\rangle)\right]=0 .
$$

Assume the cost function

$$
\begin{aligned}
f(H,\langle u\rangle) & =f_{1}(H)+\left\langle u^{T} r u\right\rangle, \\
f_{1}(H) & =s_{0}+s_{1} H+s_{2} H^{2}+s_{3} H^{3},
\end{aligned}
$$

where $r$ is a constant. Thus, the optimal control force is determined:

$$
u^{*}=\frac{1}{2 r} \frac{\partial V}{\partial H}\left(\frac{\partial H}{\partial P_{1}}-\frac{\partial H}{\partial P_{2}}\right)
$$

Replace $u$ with $u^{*}$ in equation (19) and finish the averaging to obtain final dynamical programming equation. Then, $(\partial V / \partial H)$ can be obtained by solving this final dynamical programming equation. Substituting $(\partial V / \partial H)$ into equation (22) and replacing $u$ with optimal control force $u^{*}$ in equation (11), the fully averaged Itô equation is

$$
\mathrm{d} H=\overline{\bar{m}}(H) \mathrm{d} t+\bar{\sigma}(H) \mathrm{d} B(t),
$$

$$
\overline{\bar{m}}(H)=\bar{m}(H)+\left\langle u^{*}\left(-\frac{\partial H}{\partial P_{1}}+\frac{\partial H}{\partial P_{2}}\right)\right\rangle .
$$

According to the theory of stochastic dynamics, $H$ is a Markov diffusion process, and the transition probability density function is satisfied by the so-called FokkerPlanck-Kolmogorov (FPK) equation. Solving the FPK equation yields the following stationary probability density of $H(t)$ :

$$
p^{c}(H)=C^{c} \exp \left\{-\int_{0}^{H}\left[\frac{\left.\left(-2 \overline{\bar{m}}(u)+\mathrm{d} \bar{\sigma}^{2}(u)\right) / \mathrm{d} u\right]}{\bar{\sigma}^{2}(u) \mathrm{d} u}\right\} .\right.
$$

The stationary joint probability densities $p^{c}(\mathbf{q}, \mathbf{p})$ and mean square value $E\left[Q_{1}^{2}\right], E\left[Q_{2}^{2}\right]$ are then obtained as follows [14]:

$$
\begin{aligned}
p(\mathbf{q}, \mathbf{p}) & =\left.\frac{p^{c}(H)}{T(H)}\right|_{H=H(\mathbf{q}, \mathbf{p})}, \\
E\left[Q_{i}^{2}\right] & =\int_{-\infty}^{\infty} q_{i}^{2} p(\mathbf{q}, \mathbf{p}) \mathrm{d} q_{1} \mathrm{~d} q_{2} \mathrm{~d} p_{1} \mathrm{~d} p_{2}, \quad i=1,2 .
\end{aligned}
$$

Introduce control effectiveness to measure the performance of the proposed control strategy:

$$
K_{i}=\frac{E^{u}\left[Q_{i}^{2}\right]-E^{c}\left[Q_{i}^{2}\right]}{E^{u}\left[Q_{i}^{2}\right]}, \quad i=1,2,
$$

where 


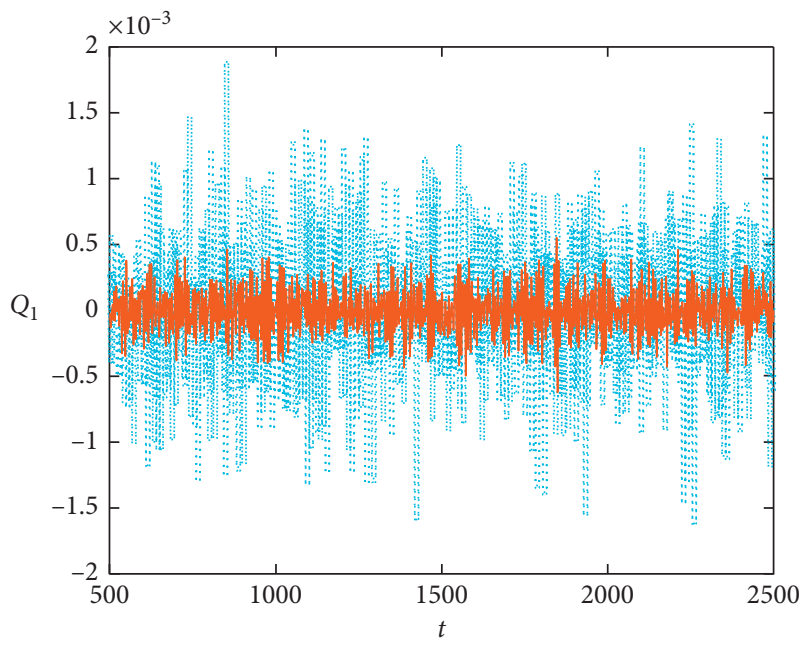

..... Uncontrolled

— Controlled

Figure 4: Sample of process $q_{1}$ of system (10).

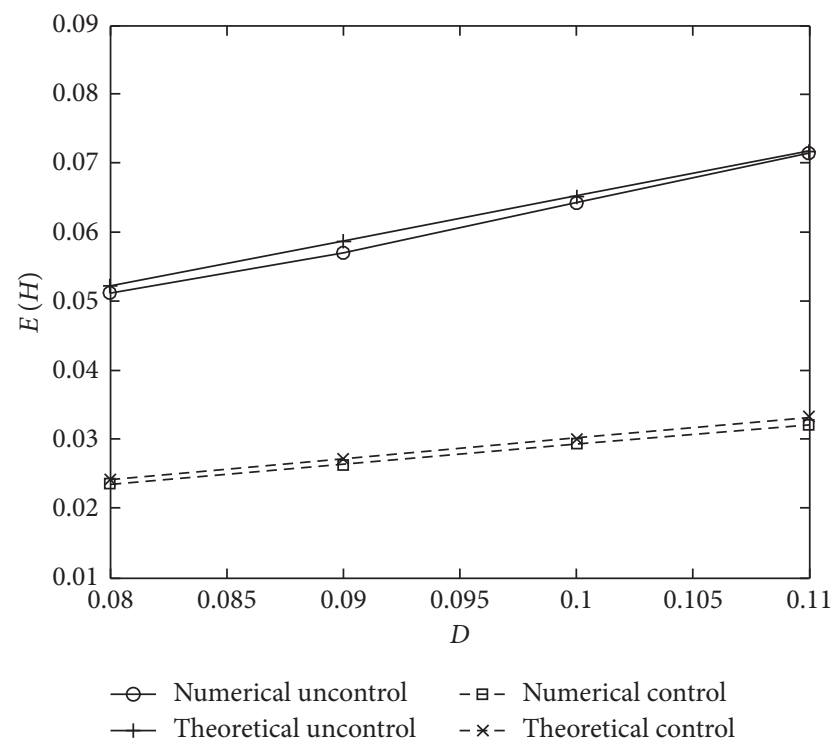

Figure 5: Mean value $E[H]$ of system (10) as function of $D$.

where $E^{u}\left[Q_{i}^{2}\right]$ and $E^{c}\left[Q_{i}^{2}\right]$ denote uncontrolled and controlled mean square value of $Q_{i}$, respectively.

\section{Numerical Results}

As a verification method of control strategy, Monte Carlo simulation has been widely used in many research studies, which is practical and efficient. In this paper, the Monte Carlo simulation method is used, too. With specific system parameters $\quad k_{p}=10.702 \mathrm{kN} / \mathrm{m}, \quad c_{p}=28.681 \mathrm{~N} \cdot \mathrm{s} / \mathrm{m}$, $k=54.684 \mathrm{kN} / \mathrm{m}, \quad k_{3}=300 \mathrm{kN} / \mathrm{m}, \quad c=196.02 \mathrm{~N} \cdot \mathrm{s} / \mathrm{m}$, $D=0.1$, and $m=2 \mathrm{~kg}, M=10 \mathrm{~kg}$ and control parameters $s_{0}=1, s_{1}=1, s_{2}=1$, and $r=0.05$, some results for uncontrolled and optimally controlled system (4) are obtained and shown in Figures 3-7.

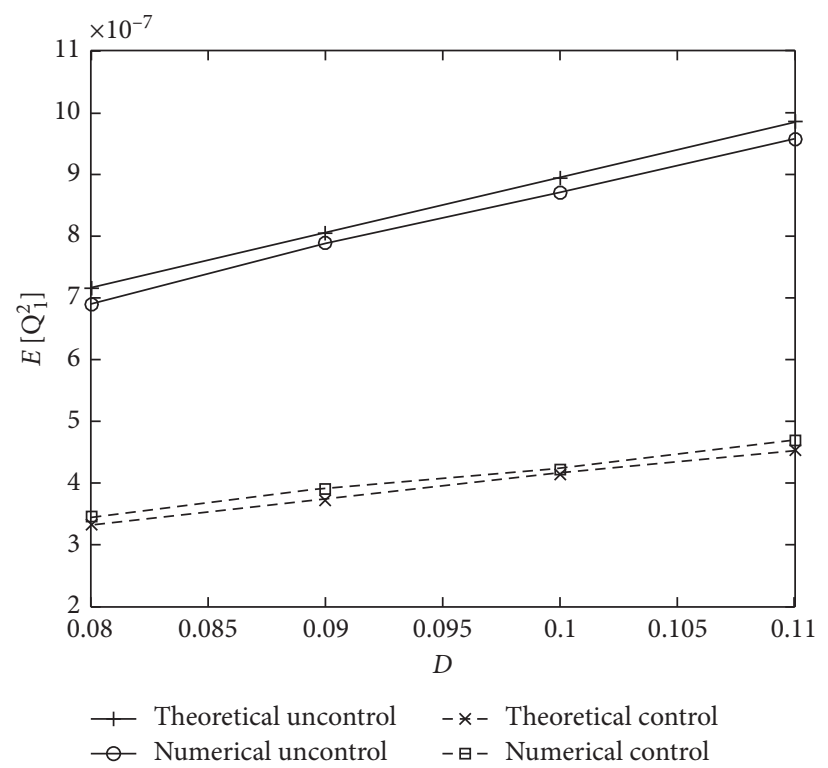

FIgUre 6: Mean square value $E\left[Q_{1}^{2}\right]$ of system (10) as function of $D$.

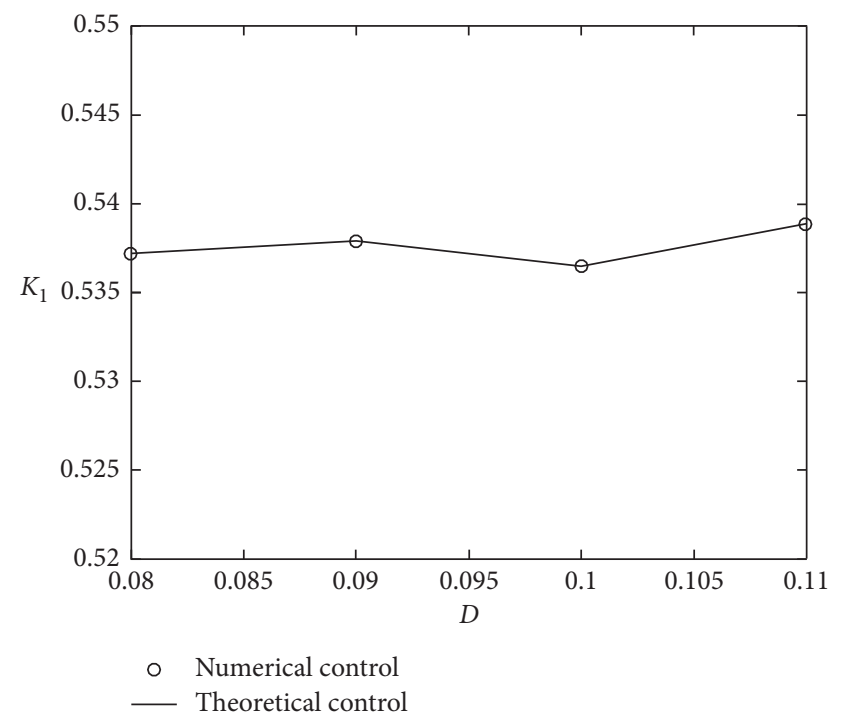

FIgURE 7: Control effectiveness $K_{1}$ of system (10) as function of $D$.

In Figure 3, the stationary probability density $p(H)$ of total energy $H$ of system (4) is plotted. It is seen that the $p(H)$ curve of the optimally controlled system shifts to the left and has higher peak value when the optimal control force is applied. This means that the structure has higher probability to vibrate in small amplitude, which indicates the proposed control strategy is very effective for response reduction. In Figure 3, the solid lines are analytical results obtained from solving equation (25) while the symbols are Monte Carlo simulation results directly obtained from equation (4). They agree well, which illustrates the accuracy of the proposed method. Figure 4 plots the samples of generalized displacement $Q_{1}$ of optimally controlled and uncontrolled system (10), from which the response reduction of our proposed method can be observed intuitively. Figures 5 and 
6 show the mean value $E[H]$ of total energy and mean square value $E\left[Q_{1}^{2}\right]$ of generalized displacement varying with the intensity of random excitation. It is seen that with the increase of the intensity of excitation, the response of the optimally controlled and uncontrolled systems increases. However, the response of the optimally controlled system is always much smaller than the uncontrolled one. Figure 7 shows the control effectiveness $K_{1}$ varying with the intensity of the excitation. With different intensities of excitation, control effectiveness changes smoothly between 53\%-54\%, which indicates this control strategy has good robustness. Compared with existing literatures, the control effectiveness of this control strategy using the piezoelectric stack inertial actuator is much higher, for example, in ref. [7], it can be seen from the figure of vibration response for simultaneous control of multiple harmonics that the control effectiveness is about $10 \%-30 \%$. In ref. [8], it can be seen from the figure of comparison of the plate vibrations in the frequency domain without control and with control that the control effectiveness is about $10 \%-35 \%$.

\section{Conclusion}

An optimal control strategy for nonlinear stochastic vibration using a piezoelectric stack inertial actuator has been proposed in this paper. This proposed procedure has some advantages: the control problem is investigated in the Hamiltonian frame, which makes the stochastic averaging method for quasi-Hamiltonian system available for dimension reduction; the proposed control law is analytical and can be fully executed by a piezoelectric stack inertial actuator. Numerical results showed that the strategy is fairly robust and effective in reduction of stationary response of the controlled system by using piezoelectric stack inertial actuator; compared with those in some literatures, this proposed control strategy has higher effectiveness. The main work of our further research is to use the theoretical advantage of this method to specific experiments. Thus, it is potentially promising for practical control applications after further research.

\section{Data Availability}

The data used to support the findings of this study are available from the corresponding author upon request.

\section{Conflicts of Interest}

The authors declare that there are no conflicts of interest.

\section{Acknowledgments}

The study was supported by National Key R\&D Program of China (Grant no. 2018YFC0809400) and National Natural Science Foundation of China (Grant no. 11802267)

\section{References}

[1] H. Elahi, M. Eugeni, P. Gaudenzi, F. Qayyum, R. F. Swati, and H. M. Khan, "Response of piezoelectric materials on thermomechanical shocking and electrical shocking for aerospace applications," Microsystem Technologies, vol. 24, no. 9, pp. 3791-3798, 2018.

[2] L. Song and P. Xia, "Active control of helicopter structural response using piezoelectric stack actuators," Journal of Aircraft, vol. 50, no. 2, pp. 659-663, 2013.

[3] H. P. Syahputra, T. J. Ko, and B. M. Chung, "Development of 2-axis hybrid positioning system for precision contouring on micro-milling operation," Journal of Mechanical Science and Technology, vol. 28, no. 2, pp. 691-697, 2014.

[4] J. Guo, S. K. Chee, T. Yano, and T. Higuchi, "Micro-vibration stage using piezo actuators," Sensors and Actuators A: Physical, vol. 194, no. 5, pp. 119-127, 2013.

[5] L. Benassi, S. J. Elliott, and P. Gardonio, "Active vibration isolation using an inertial actuator with local force feedback control," Journal of Sound and Vibration, vol. 276, no. 1-2, pp. 157-179, 2004.

[6] S. B. Choi, S. R. Hong, and Y. M. Han, "Dynamic characteristics of inertial actuator featuring piezoelectric materials: experimental verification," Journal of Sound and Vibration, vol. 302, no. 4-5, pp. 1048-1056, 2007.

[7] M. Li, T. C. Lim, W. S. Shepard Jr., and Y. H. Guan, "Experimental active vibration control of gear mesh harmonics in a power recirculation gearbox system using a piezoelectric stack actuator," Smart Materials and Structures, vol. 14, no. 5, pp. 917-927, 2005.

[8] G. Zhao, N. Alujević, B. Depraetere, G. Pinte, J. Swevers, and P. Sas, "Experimental study on active structural acoustic control of rotating machinery using rotating piezo-based inertial actuators," Journal of Sound and Vibration, vol. 348, pp. 15-30, 2015.

[9] Y. Shao, S. B. Shao, M. L. Xu, S. Y. Song, and Z. Tian, “An inertial piezoelectric actuator with miniaturized structure and improved load capacity," Smart Materials and Structures, vol. 28 , no. 5, p. 55023, 2019.

[10] R. Wang, Y. Hu, D. Shen, J. Ma, J. Wen, and J. Li, “Design and experimental performance of a novel piezoelectric inertial actuator for magnetorheological fluid control using permanent magnet,” IEEE Access, vol. 7, pp. 43573-43580, 2019.

[11] P. Konstanzer, P. Jänker, and S. Storm, "A piezo inertial force generator optimized for high force and low frequency," Smart Materials and Structures, vol. 16, no. 4, pp. 1260-1264, 2007.

[12] E. Lu, W. Li, X. Yang, Y. Wang, and Y. Liu, "Optimal placement and active vibration control for piezoelectric smart flexible manipulators using modal $\mathrm{H}_{2}$ norm," Journal of Intelligent Material Systems and Structures, vol. 29, no. 11, pp. 2333-2343, 2018.

[13] S.-B. Choi and S.-R. Hong, "Active vibration control of a flexible structure using an inertial type piezoelectric mount," Smart Materials and Structures, vol. 16, no. 1, pp. 25-35, 2006.

[14] W. Q. Zhu and Y. Q. Yang, "Stochastic averaging of quasinonintegrable-Hamiltonian systems," Journal of Applied Mechanics, vol. 64, no. 1, pp. 157-164, 1997.

[15] W. Zhu and Z. Ying, "Optimal nonlinear feedback control of quasi-Hamiltonian systems," Science in China Series A: Mathematics, vol. 42, no. 11, pp. 1213-1219, 1999. 